\title{
Home-based Screening for Obstructive Sleep Apnea in Children
}

\author{
Dylan Bertoni ${ }^{1}$ and Amal Isaiah ${ }^{1,2}$ \\ 1. Department of Otorhinolaryngology_Head and Neck Surgery, University of Maryland School of Medicine, Baltimore, MD, USA; \\ 2. Department of Pediatrics, University of Maryland School of Medicine, Baltimore, MD, USA
}

DOI: https://doi.org/10.17925/USPRD.2020.5.1.38

$\mathrm{P}$ ediatric obstructive sleep apnea (OSA) is the most severe form of sleep disordered breathing, characterized by repeated airway obstruction and desaturation during sleep. The current diagnostic gold standard is overnight polysomnography (PSG), which stratifies the severity of OSA based on the apnea hypopnea index (AHI). The first line of treatment is tonsillectomy and adenoidectomy, which leads to the resolution, or the improvement of, the condition in the majority of the children. Children with severe OSA (defined as AHI >10) are at an elevated risk of perioperative adverse events, and are typically monitored overnight following tonsillectomy and adenoidectomy. However, only a small number of children undergo a preoperative PSG due to the high cost, and relatively low number, of certified sleep centers. Thus, there is a strong need for an improved screening pathway for children with OSA. While home sleep apnea testing (HSAT) has become central to the diagnosis of OSA in adults, there is a paucity of data validating the use of HSAT in children. The purpose of this review is to highlight and examine recent developments in physiological monitoring that could improve the diagnostic and screening strategies for pediatric OSA.

\section{Keywords}

Pediatric obstructive sleep apnea, polysomnography, home sleep apnea testing

Disclosures: Amal Isaiah receives patent-related royalties related to inventions in sleep apnea imaging not discussed in the current paper. Dylan Bertoni has no financial or non-financial relationships or activities to declare in relation to this article.

Review Process: Double-blind peer review.

Compliance with Ethics: This article involves a review of the literature and did not involve any studies with human or animal subjects performed by either of the authors.

Authorship: The named authors meet the International Committee of Medical Journal Editors (ICMJE) criteria for authorship of this manuscript, take responsibility for the integrity of the work as a whole, and have given final approval for the version to be published.

Access: This article is freely accessible at touchRESPIRATORY.com (c) Touch Medical Media 2020.

Received: April 14, 2020

Accepted: May 11, 2020

Published Online: December 23, 2020

Citation: US Respiratory \& Pulmonary Diseases. 2020; 5(1):38-42

Corresponding Author: Amal Isaiah,

Deptartment of Otorhinolaryngology-Head and Neck

Surgery, University of Maryland School of Medicine,

16 S Eutaw St, Suite 500, Baltimore, MD 21201, USA.

E: aisaiah@som.umaryland.edu

Support: No funding was received in

the publication of this article.
Sleep disordered breathing in children comprises a spectrum of abnormal breathing patterns associated with increased airflow resistance and sleep disruption. Sleep disordered breathing is categorized by severity into primary snoring, upper airway resistance syndrome, and obstructive sleep apnea (OSA). Pediatric OSA has a prevalence of up to $5 \%$ in children.' The most common cause of increased upper airway resistance and pharyngeal collapse is attributed to enlarged lymphoid tissue within the tonsils and adenoids. ${ }^{2}$ Additional risk factors include obesity, ${ }_{1}^{3}$ and neuromuscular ${ }^{4}$ or craniofacial disorders. ${ }^{5}$ The most common symptoms reported by parents of children with OSA are snoring and difficulty breathing during sleep. Other nighttime symptoms include gasping, restless sleep, frequent awakenings, enuresis, and sweating. ${ }^{6}$ During the daytime, parents may also report difficulty waking the child and excess sleepiness, but children more commonly experience behavioral impairments such as aggression, impulsivity, and hyperactivity, ${ }^{7.8}$ or neurocognitive impairments such as deficits in attention, executive function, and language." Untreated OSA has also been associated with detrimental effects on growth and development, ${ }^{10}$ memory, ${ }_{11}^{11}$ and quality of life ${ }^{12}$ in children, therefore supporting its prompt diagnosis and management.

Pediatric OSA is diagnosed and stratified by standard overnight polysomnography (PSG). ${ }^{13}$ This is performed at certified sleep centers, and is scored by trained technicians according to guidelines published by the American Academy of Sleep Medicine (AASM). ${ }^{14}$ PSG measures the following during sleep: sleep stage, cardiac rhythm, oxygen saturation, muscle tone, body position, movement, and airflow. Currently, the AASM ${ }^{14}$ and the American Academy of Pediatrics ${ }^{15}$ recommend PSG for children with symptoms of sleep disordered breathing, while the American Academy of Otolaryngology-Head and Neck Surgery (AAO-HNS) recommends the screening of high-risk children. ${ }^{16}$ The severity of OSA is scored using the apnea hypopnea index (AHI), defined as the frequency of partial or complete reduction in airflow per hour. The most common classification system categorizes OSA as mild, moderate, and severe, based on the AHI thresholds of 1, 5, and 10 events per hour, respectively.12

The treatment of OSA depends on the severity and etiology of the obstruction. The standard of treatment is tonsillectomy and adenoidectomy, which leads to the resolution, or improvement, of the condition in the majority of children. ${ }^{15}$ Mild OSA may sometimes be managed with pharmacotherapy, such as intranasal steroids, to reduce upper airway inflammation. ${ }^{17}$ In children with persistent OSA after tonsillectomy and adenoidectomy, or in children with other complex comorbidities, additional surgery or treatment, such as continuous positive airway pressure (CPAP), may play a role..$^{18-22}$ While CPAP is the standard of treatment of OSA in adults, non-compliance observed in children negatively impacts the management of persistent OSA. ${ }^{23}$ 
Table 1: Summary of differences between polysomnography and home sleep apnea testing

\begin{tabular}{l|l|l|}
\hline Metric & PSG & HSAT \\
\hline Cost & High $(\$ 1,000-4,000)$ & Low* \\
\hline Convenience & Inconvenient/cumbersome & Convenient/easy to use \\
\hline Safety & Safe & Safe \\
\hline Accessibility & Difficult to access/schedule, long wait times & Easily accessible \\
\hline Personnel & Additional technicians needed for implementation/interpretation & No additional personnel required \\
\hline Diagnostics & Gold-standard for diagnosis, reference for sensitivity/specificity/accuracy & Not clinically validated, lower sensitivity/specificity/accuracy \\
\hline
\end{tabular}

*Relative to standard PSG, cost savings between $22 \%$ and $42 \%$ have been reported with HSAT use in adults with OSA. Downstream costs of a $12 \%$ HSAT failure rate resulting in PSG were not included. HSAT = home sleep apnea testing; OSA = obstructive sleep apnea; PSG = polysomnography.

Due to the increased risk of perioperative respiratory complications associated with anesthesia, children with severe OSA defined by $\mathrm{AHI} \geq 10$ are observed overnight following surgery, ${ }^{16}$ which requires a preoperative PSG that costs $\$ 1,000-4,000.24,25$ Furthermore, the inadequate number of sleep laboratories and trained personnel may lead to long wait times in some areas, which partially explains the preoperative utilization of PSG remaining below $10 \%{ }^{26}$ Clinical parameters, such as parental history and physical examination findings are poor predictors of the presence and/or severity of OSA. ${ }^{27,28}$ Furthermore, seemingly healthy children could be diagnosed with very severe OSA, ${ }^{29}$ and treatment-related changes in OSA outcomes are not causally attributable to changes in OSA severity as measured by PSG, supporting an improved screening pathway for children with OSA. ${ }^{30}$

Home sleep apnea testing (HSAT) involves the use of portable or wearable sensors during sleep at home to screen for OSA with or without a PSG. Generally, HSAT requires significantly fewer resources and may provide clinical benefit at a lower cost. ${ }^{31}$ However, the AASM does not support the universal use of HSAT in children due to lack of data supporting its validity in an unsupervised environment. ${ }^{31}$ Furthermore, the application of probes with cables in children may pose a hazard. Finally, the use of AHI as an objective parameter to define treatment candidacy, as well as to measure the efficacy of its treatment, places a major barrier for its expanded use as a comparable metric has not been defined in this population. The purpose of this review is to highlight and examine some of the recent strategies being proposed as potential screening or diagnostic tools for pediatric OSA, and to emphasize the need for further research to validate the use of HSAT.

\section{Screening technologies}

The central theme of HSAT is to replicate the accuracy of in-lab testing while remaining safe and easy-to-use at home. This is commonly achieved by specific polysomnographic channels or their combinations. These signals can be separated by domains of interest according to the Sleep, Cardiovascular, Oximetry, Position, Effort, and Respiratory (SCOPER) acronym. ${ }^{32,33}$ Each section of this review will address these domains and their feasibility as HSAT for children, either on their own or as a combination, instead of a regular, overnight, laboratory-based PSG. A summary of key differences between PSG and HSAT is shown in Table 1.

\section{Sleep}

Standard PSG combines electroencephalography (EEG) with electromyography and electrooculography to determine sleep stage and distinguish between asleep and awake states. The electrical activity measured by leads attached to the scalp, chin/neck musculature, and around the eyes, is scored by a technician. ${ }^{34}$ While home EEG recordings are technically possible, the need for application of the conducting gel to reduce impedance levels and the potential for injury from loose wires are likely to prevent its widespread use. ${ }^{35}$ Collop et al. ${ }^{32}$ recommend the inclusion of sensors in the sleep domain, because ideally the ultimate index used to assess the severity of sleep disordered breathing will depend upon the amount of time spent asleep, rather than the amount of time spent recording. Alternative options for assessing sleep/wake states include video monitoring ${ }^{3,37}$ and actigraphy. Actigraphy uses accelerometers to measure movement data. Clinically validated actigraphs are useful for the estimation of parameters such as the total sleep time and sleep efficiency. ${ }^{38-40}$ Actigraphy could be used to estimate sleep/wake states when PSG is unavailable..$^{41,42}$ In other studies, a polyvinylidene difluoride (PVDF) sensor in bedsheets, when combined with wrist actigraphy, detected sleep/wake states in up to $90 \%$ of the instances when compared to PSG. Furthermore, the heart rate and respiratory rate measured by the sheet sensor had overall accuracy exceeding 95\%. ${ }^{43}$

Sleep quality has also been assessed with clinically validated parental questionnaires such as the Pediatric Sleep Questionnaire, ${ }^{44}$ the Epworth Sleepiness Scale, ${ }^{45}$ the OSA Score, ${ }^{46}$ and the OSA-18. ${ }^{47}$ These questionnaires have shown mixed results with predicting the presence of OSA, and cannot accurately determine OSA severity. ${ }^{28}$ However, eliminating redundant items in these questionnaires has been shown to improve their predictive performance for OSA severity, ${ }^{48}$ and data mining and machine learning algorithms may also improve their accuracy. ${ }^{49}$

\section{Cardiovascular}

The chronic airflow obstruction and hypoxemia that occur in children with OSA have been associated with detrimental effects on the heart, such as right and left ventricular dysfunction, ${ }^{50}$ elevated blood pressure, ${ }^{51}$ and autonomic dysfunction. ${ }^{52}$ The long-term effects of OSA on cardiac health are not well established, ${ }^{53}$ and preoperative assessments of cardiac function using echocardiography or electrical activity by electrocardiography are not associated with risk reduction. ${ }^{54,55}$ Heart rate variability is a measurement of these inter-beat variations in cardiac rhythm and has been shown to change predictably among children with OSA..$^{56}$ Furthermore, treatment of OSA with tonsillectomy and adenoidectomy has been shown to significantly change heart rate variability parameters, although these changes were not found to be causally mediated by changes in AHI. ${ }^{57}$ Signal analysis of an at-home electrocardiogram (ECG) could be adopted as a screening tool for children with OSA. ${ }^{58,59}$ 
Peripheral arterial tonometry (PAT) measures changes in pulse wave amplitude in the finger, representing sympathetic activation in the vasculature resulting from changes in local oxygen levels. In adults, HSAT with PAT has been shown to predict PSG parameters with acceptable accuracy. ${ }^{60}$ The Watch-PAT ${ }^{\circledast}$ (Itamar Medical, Tel-Aviv, Israel) uses both PAT and actigraphy. The Watch-PAT demonstrated very high correlation with $\mathrm{AHI}$ in adults, ${ }^{61}$ as well as in adolescents; ${ }^{62}$ although its accuracy was only $61 \%$ in younger children. ${ }^{63}$ The sensitivity and specificity of the Watch-PAT vary depending on the threshold set for detecting AHI. ${ }^{64,65}$ Unfortunately, the algorithm used in Watch-PAT is proprietary. Furthermore, the physiology of the autonomic nervous system may change with age, and therefore cannot be generalized for use in all age groups. ${ }^{66}$

\section{Oximetry}

Pulse oximetry uses red or infrared light passed through a finger-tip, toe-tip, or earlobe, to measure the amount of oxygen carried by hemoglobin. ${ }^{67}$ Airflow obstruction is associated with peripheral hypoxia, which is detected using oximetry. Overnight oximetry curves alone could be used to identify OSA with a positive predictive value of $97 \%$, and an inter-observer agreement of $80 \%{ }^{68}$ However, when these criteria were used in obese children, the sensitivity and specificity were $58 \%$ and $88 \%$, respectively, and the positive predictive value dropped to $69 \% .{ }^{69}$ Wearable devices such as the Pulse Oximetry Watch (CloudCare HealthCare Ltd, Chengdu, China) demonstrated poor sensitivities and specificities $(<75 \%)$ with an $\mathrm{AHI}$ threshold of $\leq 15 .^{70}$ Phone-based oximeters that utilize the phone camera flash and lens with an external plug-in probe can effectively measure oximetry in pediatric patients, ${ }^{71}$ and analysis of these recordings yielded an accuracy of $87 \%$, a sensitivity of $80 \%$, and a specificity of $92 \%{ }^{72}$ While oximetry data from a controlled setting can provide useful information, the feasibility of oximetry at home raises concern as parental experience may impact data collection. ${ }^{73}$ Night-to-night at-home recordings of pulse oximetry were highly consistent with correlations of $90 \%$, although variability increased with younger children. ${ }^{74}$ Difficulties with home oximetry recordings could be overcome by recording over multiple nights.

\section{Position}

Sleep-related arousals are a component of the definition of a hypopnea, and therefore contribute to the severity of OSA determined by the AHI. Conventional PSG utilizes body position sensors to track movements and changes in position following arousals. ${ }^{34}$ Body movement is greatly reduced or eliminated during deep stage 3 and rapid eye movement (REM) sleep relative to lighter sleep stages and awake states, which facilitates identification of the sleep stage. Examples of clinically validated actigraphs include the Motionlogger ${ }^{\circledR}$ Sleep Watch (Ambulatory Monitoring, Ardsley, NY, USA) and Actiwatch- $2^{\circledR}$ (Philips Respironics, Amsterdam, The Netherlands). Actigraphy can achieve reasonably high sensitivity (88-92\%) and accuracy (84-86\%), but is limited by low specificity (46-66\%). ${ }^{75,76}$ While actigraphy is limited in its ability to predict OSA, it is effective at evaluating sleep/wake states in children. ${ }^{77}$ Commercial devices and smartphone applications are associated with poor accuracy.76,78 Other position sensors include in-bed sensors, in-pillow sensors, and belts placed around the body. ${ }^{79,80}$

\section{Effort}

The gold standard for effort measurement, which is central to PSG, is esophageal manometry. Due to its invasive nature, it has been replaced by belt sensors utilizing piezoelectric, polyvinylidene fluoride (PVDF), or respiratory inductance plethysmography (RIP). The sensors in piezoelectric or PVDF belts are located in a portion of the strap and are dependent on the transmission of force along the belt, whereas the entire RIP belt is the sensor. ${ }^{81}$ The AASM recommends either dual RIP belts, dual PVDF belts, or a combination of the two for detection of respiratory effort. ${ }^{34}$ These belt sensors are comfortable and could be implemented in an in-home setting. Dual RIP belts have been combined with oximetry to develop a screening tool for pediatric OSA, with a correct classification rate of $83 \%{ }^{82}$ Recently, PVDF sensors have been compared to RIP in children, finding no significant differences in detecting respiratory events. ${ }^{83}$ Additionally, suprasternal pressure sensors, such as the PneaVoX (Cidelec, Sainte Gemmes sur Loire, France), have been developed and compared with standard RIP for detection of apneas. Sensitivity ranged from $98-100 \%$, and specificity ranged from $70-75 \%$, with a significant number of RIP-determined central apneas scored as obstructive apneas by PneaVoX. ${ }^{84}$

\section{Respiratory}

The AASM defines an obstructive apnea as a decrease in oronasal airflow by $90 \%$ or more, and a hypopneic event is defined as a decrease in oronasal airflow by $\geq 50 \%$ in the presence of ongoing respiratory effort for two respiratory cycles. ${ }^{34}$ Given that the reduction in oronasal airflow is central to the definitions of both apneas and hypopneas, airflow measurement devices could potentially be used during HSAT. The principal limitation of nasal pressure transducers is the discomfort experienced by the patient over the course of the night. The Flow Wizard ${ }^{\circledast}$ (DiagnoselT, Sydney, Australia) is a single-channel nasal airflow pressure transducer to screen for OSA. The accuracy of the Flow Wizard increased from $84 \%$ in adult patients with $\mathrm{AHI}>5$ to $96 \%$ in patients with $\mathrm{AHI}>30$. On average, the $\mathrm{AHI}$ determined by the Flow Wizard was 6.2 events/hour lower than the standard PSG. ${ }^{85}$ The ApneaLink ${ }^{\mathrm{TM}}$ Plus (ResMed, San Diego, CA, USA) also measures airflow in addition to oximetry, respiratory effort, and heart rate. In a study of obese adolescents with mild, moderate, and severe OSA, the sensitivity associated with the use of ApneaLink Plus ranged from 85-100\%, and specificity ranged from 46-90\%. ${ }^{86}$ Using logistic regression to model home airflow recordings combined with oximetry had an accuracy of $86.3 \%$, a positive predictive value of $88.4 \%$, and an area under receiver operator characteristic curve of 0.95 , demonstrating an excellent overall predictive capability. ${ }^{87}$

\section{Conclusions}

Although fixed anesthesia protocols may be able to mitigate the perioperative risk associated with OSA, ${ }^{88}$ preoperative risk stratification is necessary for safely performing tonsillectomy and adenoidectomy. The challenges of cost and accessibility warrant continued investigation into alternatives to a PSG. The AASM maintains a position that there is insufficient data to support the current use of HSAT in children. The validation of HSAT in children is limited by the perceived need to achieve results identical to a PSG. Using the AHI as the sole metric for determining resolution of OSA following tonsillectomy and adenoidectomy also precludes the widespread implementation of HSAT in children. That said, PSG is a test of cardiopulmonary variation during sleep and does not predict morbidity in every instance. ${ }^{30,89}$ Clinical parameters are poor predictors of OSA severity, yet a majority of children undergo surgery without a PSG. Furthermore, no outcome-related differences have been 
found in these children relative to those who obtain pre-treatment screening or diagnosis with PSG. ${ }^{26}$ Many of the technologies mentioned in this review unfortunately do not achieve accuracies, sensitivities, or specificities that can be considered equivalent to those of PSG. Additionally, HSAT is unsuitable for the diagnosis of pediatric sleep disorders, such as central sleep apnea, periodic limb movement disorder, and parasomnias, which require video recording and therefore a full PSG.
Currently, HSAT-based testing is the diagnostic standard for adults with OSA. The transition from in-lab PSG to HSAT in the adult population required substantial research into feasibility and cost effectiveness, which could not be replicated in children. The number of children requiring diagnostic evaluation for OSA is also likely to increase with the rising burden of obesity. In an effort to refine healthcare delivery and to contain the costs associated with the screening process, this is a domain that merits further research. $\square$

\section{Article highlights}

- Pediatric obstructive sleep apnea has a prevalence of 5\%; it is diagnosed by overnight polysomnography and treated with tonsillectomy and adenoidectomy

- Preoperative risk stratification is important to recognize children at risk of perioperative adverse events

- Preoperative polysomnography for all children is not feasible due to the high cost and inadequate number of certified sleep centers

- Home sleep apnea testing is used to diagnose obstructive sleep apnea in adults, but is not approved for use in children due to a lack of data for validation

- A number of sensors and technologies have been developed for data collection in SCOPER domains (Sleep, Cardiovascular, Oximetry, Position, Effort, and Respiratory)

- Further research into these potential screening strategies is warranted to reduce costs associated with diagnosing obstructive sleep apnea, and to improve perioperative safety in these children

1. Lumeng JC, Chervin RD. Epidemiology of pediatric obstructive sleep apnea. Proc Am Thorac SOC. 2008:5:242-52.

2. Marcus $\mathrm{CL}$, Loughlin GM. Obstructive sleep apnea in children. Semin Pediatr Neurol. 1996:3:23-8.

3. Mitchell RB, Kelly J. Outcome of adenotonsillectomy for obstructive sleep apnea in obese and normal-weight children Otolaryngol Head Neck Surg. 2007;137:43-8.

4. Katz SL. Assessment of sleep-disordered breathing in pediatric neuromuscular diseases. Pediatrics. 2009;123(Suppl. 4):S222.

5. Cielo CM, Marcus CL. Obstructive sleep apnoea in children with craniofacial syndromes. Paediatr Respir Rev. 2015;16:189-96.

6. Bitners AC, Arens R. Evaluation and management of children with obstructive sleep apnea syndrome. Lung. 2020;198:257-70.

7. Perfect MM, Archbold K, Goodwin JL, et al. Risk of behavioral and adaptive functioning difficulties in youth with previous and current sleep disordered breathing. Sleep. 2013;36:517-25.

8. Landau YE, Bar-Yishay O, Greenberg-Dotan S, et al. Impaired behavioral and neurocognitive function in preschool children with obstructive sleep apnea. Pediatr Pulmonol. 2012;47:180-8.

9. O'Brien LM, Mervis $\mathrm{CB}$, Holbrook CR, et al. Neurobehavioral correlates of sleep-disordered breathing in children. J Sleep Res. 2004:13:165-72.

10. Nieminen P, Tolonen U, Lopponen H. Snoring and obstructive sleep apnea in children: a 6-month follow-up study. Arch Otolaryngol Head Neck Surg. 2000;126:481-6.

11. Cha J, Zea-Hernandez JA, Sin S, et al. The effects of obstructive sleep apnea syndrome on the dentate gyrus and learning and memory in children. J Neurosci. 2017:37:4280-8

12. Marcus $\mathrm{CL}$, Moore RH, Rosen $\mathrm{CL}$, et al. A randomized trial of adenotonsillectomy for childhood sleep apnea. N Eng/ J Med. 2013;368:2366-76

13. Schechter MS. Technical report: diagnosis and management of childhood obstructive sleep apnea syndrome. Pediatrics. 2002;109:e69.

14. Aurora RN, Zak RS, Karippot A, et al. Practice parameters for the respiratory indications for polysomnography in children. Sleep. 2011;34:379-88.

15. Marcus $C L$, Brooks $\sqcup$, Ward $S D$, et al. Diagnosis and management of childhood obstructive sleep apnea syndrome. 2012;130:e714-55

16. Roland PS, Rosenfeld RM, Brooks $\sqcup$, et al. Clinical practice guideline: polysomnography for sleep-disordered breathing prior to tonsillectomy in children. Otolaryngol Head Neck Surg. 2011;145(Suppl. 1):S1-15

17. Kheirandish-Gozal L, Bhattacharjee R, Bandla HPR, Gozal D. Antiinflammatory therapy outcomes for mild OSA in children. Chest. 2014;146:88-95.

18. Isaiah A, Kiss E, Olomu P, et al. Characterization of upper airway obstruction using cine MRI in children with residual obstructive sleep apnea after adenotonsillectomy. Sleep Med. 2018;50:79-86.

19. Pirelli P, Saponara M, Guilleminault C. Rapid maxillary expansion in children with obstructive sleep apnea syndrome. Sleep. 2004;27:761-6.

20. Rizzi CJ, Amin JD, Isaiah A, et al. Tracheostomy for severe pediatric obstructive sleep apnea: indications and outcomes. Otolaryngol Neck Surg. 2017:157:309-13.

21. Shott SR. Evaluation and management of pediatric obstructive sleep apnea beyond tonsillectomy and adenoidectomy. Curr Opin otolaryngol Head Neck Surg. 2011:19:449-54.

22. Manickam PV, Shott SR, BosS EF, et al. Systematic review of site of obstruction identification and non-CPAP treatment options for children with persistent pediatric obstructive sleep apnea. Laryngoscope. 2016:126:491-500.

23. Marcus CL, Ward SLD, Mallory GB, et al. Use of nasal continuous positive airway pressure as treatment of childhood obstructive sleep apnea.J Pediatr. 1995;127:88-94.

24. Horwood L, Brouillette RT, MCGregor CD, et al. Testing for pediatric obstructive sleep apnea when health care resources are rationed. JAMA Otolaryngol Head Neck Surg. 2014;140:616-23.

25. Mitchell M, Werkhaven JA. Cost-effectiveness of polysomnography in the management of pediatric obstructive sleep apnea. Int J Pediatr Otorhinolaryngol. 2020:133:109943.

26. Mitchell RB, Pereira KD, Friedman NR. Sleep-disordered breathing in children: survey of current practice. Laryngoscope. 2006;116:956-8.

27. Wang RC, Elkins TP, Keech D, et al. Accuracy of clinical evaluation in pediatric obstructive sleep apnea. Otolaryngol Neck Surg. 1998;118:69-73.

28. Mitchell RB, Garetz S, Moore RH, et al. The use of clinical parameters to predict obstructive sleep apnea syndrome severity in children: the Childhood Adenotonsillectomy (CHAT) study randomized clinical trial. JAMA Otolaryngol Head Neck Surg. 2015;141:130-6.

29. Isaiah A, Hamdan $H$, Johnson RF, et al. very severe obstructive sleep apnea in children: outcomes of adenotonsillectomy and ris factors for persistence. Otolaryngol Neck Surg. 2017:157:128-34.

30. Isaiah A, Pereira KD, Das G. Polysomnography and treatment-related outcomes of childhood sleep apnea. Pediatrics. 2019:144:e20191097.

31. Kirk V, Baughn J, D'Andrea L, et al. American academy of sleep medicine position paper for the use of a home sleep apnea test for the diagnosis of OSA in children. J Clin Sleep Med. 2017;13:1199-203.

32. Collop NA, Tracy SL, Kapur V, et al. Obstructive sleep apnea devices for out-of-center (OOC) testing: technology evaluation. J Clin Sleep Med. 2011:7:531-48.

33. Bertoni $\mathrm{D}$, Isaiah $\mathrm{A}$. Towards patient-centered diagnosis of pediatric obstructive sleep apnea-a review of biomedical engineering strategies. Expert Rev Med Devices. 2019;16:617-29.

34. Berry RB, Budhiraja R, Gottlieb DJ, et al. Rules for scoring respiratory events in sleep: update of the 2007 AASM manual for the scoring of sleep and associated events. Deliberations of the Sleep Apnea Definitions Task Force of the American Academy of Sleep Medicine. J Clin Sleep Med. 2012;8:597-619.

35. Lopez-Gordo MA, Sanchez-Morillo D, Valle FP. Dry EEG electrodes. Sensors. 2014:14:12847-70.

36. Morielli A, Ladan S, Ducharme FM, Brouillette RT. Can sleep and wakefulness be distinguished in children by cardiorespiratory and videotape recordings? Chest. 1996;109:680-7.

37. Sivan $Y$, Kornecki A, Schonfeld T. Screening obstructive sleep apnoea syndrome by home videotape recording in children. Eur Respir J. 1996:9.2127.

38. Sadeh A, Hauri PJ, Kripke DF, Lavie P. The role of actigraphy in the evaluation of sleep disorders. Sleep. 1995:18:288-302.

39. Kushida CA, Chang A, Gadkary C, et al. Comparison of actigraphic, polysomnographic, and subjective assessment of sleep parameters in sleep-disordered patients. Sleep Med. 2001;2:389-96.

40. Hedner J, Pillar G, Pittman SD, et al. A novel adaptive wrist actigraphy algorithm for sleep-wake assessment in sleep apnea patients. Sleep. 2004;27:1560-6.

41. Morgenthaler T, Alessi C, Friedman L, et al. Practice parameters for the use of actigraphy in the assessment of sleep and sleep disorders: an update for 2007. Sleep. 2007:30:519-29.

42. Hyde $\mathrm{M}, \mathrm{O}^{\prime}$ Driscoll DM, Binette $\mathrm{S}$, et al. Validation of actigraphy for determining sleep and wake in children with sleep disordered breathing. J Sleep Res. 2007;16:213-6.

43. Uchida S, Endo T, Suenaga K, et al. Sleep evaluation by a newly developed PVDF sensor non-contact sheet: a comparison with standard polysomnography and wrist actigraphy. Sleep Biol Rhythms. 2011;9:178-87.

44. Chervin RD, Weatherly RA, Garetz SL, et al. Pediatric sleep questionnaire: prediction of sleep apnea and outcomes Arch Otolaryngol Head Neck Surg. 2007;133:216-22.

45. Melendres MCS, LutZ JM, Rubin ED, Marcus CL. Daytime sleepiness and hyperactivity in children with suspected sleep-disordered breathing. Pediatrics. 2004;114:768-75.

46. Brouilette R, Hanson D, David R, et al. A diagnostic approach to suspected obstructive sleep apnea in children. J Pediatr. 1984;105:10-4.

47. Franco RAJ, Rosenfeld RM, Rao M. First place--resident clinical science award 1999. Quality of life for children with obstructive sleep apnea. Otolaryngol Head Neck Surg. 2000;123(1 Pt 1):9-16.

48. Isaiah A, Shikara M, Pereira KD, Das G. Refining screening questionnaires for prediction of sleep apnea severity in children. Sleep Breath. 2020;24:1349-56.

49. Ahmed S, Hasani S, Koone M, et al. An empirical study of questionnaires for the diagnosis of pediatric obstructive sleep apnea. Conf Proc IEEE Eng Med Biol SOC. 2018:2018:4097-100.

50. Chan JYS, Li AM, Au C-T, et al. Cardiac remodelling and dysfunction in children with obstructive sleep apnoea: a community based study. Thorax. 2009;64:233-9.

51. Kwok K, Ng DK, Chan C. Cardiovascular changes in children with snoring and obstructive sleep apnoea. Ann Acad Med Singapore. 2008;37:715-21.

52. Narkiewicz K, Montano N, Cogliati C, et al. Altered cardiovascular variability in obstructive sleep apnea. Circulation. 1998:98:1071-7.

53. Kaditis A. From obstructive sleep apnea in childhood to cardiovascular disease in adulthood: what is the evidence? Sleep 2010;33:1279-80.

54. Revenaugh PC, Chmielewski $\sqcup$, Edwards T, et al. Utility of preoperative cardiac evaluation in pediatric patients undergoing surgery for obstructive sleep apnea. Arch Otolaryngol Head Neck Surg. 2011;137:1269-75.

55. Teplitzky TB, Pereira KD, Isaiah A. Echocardiographic screening in children with very severe obstructive sleep apnea. Int J Pediatr Otorhinolaryngol. 2019;126:109626.

56. Nisbet LC, Yiallourou SR, Nixon GM, et al. Nocturnal autonomic function in preschool children with sleep-disordered breathing. Sleep Med. 2013;14:1310-6.

57. Isaiah A, Bertoni D, Pereira KD, et al. Treatment-related changes in heart rate variability in children with sleep apnea. Otolaryngol Neck Surg. 2020:162:737-45.

58. Aljadeff $\mathrm{G}$, Gozal D, Schechtman VL, et al. Heart rate variability in children with obstructive sleep apnea. Sleep. 1997;20:151-7.

59. Baharav A, Kotagal S, Rubin BK, et al. Autonomic cardiovascular control in children with obstructive sleep apnea. Clin Auton Res. 1999;9:345-51.

60. Choi JH, Kim EJ, Kim YS, et al. Validation study of portable device for the diagnosis of obstructive sleep apnea according to the 
new AASM scoring criteria: Watch-PAT 100. Acta Otolaryngol. 2010;130:838-43

61. Pittman SD, Ayas NT, MacDonald MM, et al. Using a wrist-worn device based on peripheral arterial tonometry to diagnose obstructive sleep apnea: in-laboratory and ambulatory validation Sleep. 2004;27:923-33.

62. Choi JH, Lee B, Lee JY, Kim HJ. Validating the Watch-PAT for diagnosing obstructive sleep apnea in adolescents. J Clin Sleep Med. 2018;14:1741-7.

63. Serra A, Cocuzza S, Maiolino L, et al. The Watch-PAT in pediatrics sleep disordered breathing: pilot study on children with negative nocturnal pulse oximetry. Int J Pediatr Otorhinolaryngol. 2017;97:245-50.

64. Tanphaichitr A, Thianboonsong A, Banhiran W, et al. Watch peripheral arterial tonometry in the diagnosis of pediatric obstructive sleep apnea. Otolaryngol Head Neck Surg. 2018;159:166-72

65. Su M, Yu C, Zhang Y, et al. Clinical value of portable sleep testing in children with obstructive sleep apnea syndrome. Zhonghua $E$ Ke Za Zhi. 2015;53:845-9.

66. Onder NS, Akpinar ME, Yigit O, Gor AP. Watch peripheral arterial tonometry in the diagnosis of obstructive sleep apnea: influence of aging. Laryngoscope. 2012;122:1409-14.

67. Wukitsch MW, Petterson MT, Tobler DR, Pologe JA. Pulse oximetry: analysis of theory, technology, and practice. J Clin Monit. 1988;4:290-301.

68. Brouillette RT, Morielli A, Leimanis A, et al. Nocturnal pulse oximetry as an abbreviated testing modality for pediatric obstructive sleep apnea. Pediatrics. 2000;105:405-12.

69. Van Eyck A, Lambrechts C, Vanheeswijck L, et al. The role of nocturnal pulse oximetry in the screening for obstructive sleep apnea in obese children and adolescents. Sleep Med. 2015;16:1409-12
70. Ma JR, Huang JJ, Chen Q, et al. Value of pulse oximetry watc for diagnosing pediatric obstructive sleep apnea/hypopnea syndrome. Acta Otolaryngol. 2018;138:175-9.

71. Tomlinson S, Behrmann S, Cranford J, et al. Accuracy of smartphone-based pulse oximetry compared with hospital-grade pulse oximetry in healthy children. Telemed J E Health. 2018:24:527-35.

72. Garde A, Karlen W, Dehkordi P, et al. Oxygen saturation in children with and without obstructive sleep apnea using the phone-oximeter. COnf Proc IEEE Eng Med Biol SOC. 2013;2013:2531-4.

73. Kirk VG, Bohn SG, Flemons WW, Remmers JE. Comparison of home oximetry monitoring with laboratory polysomnography in children. Chest. 2003;124:1702-8.

74. Pavone M, Cutrera R, Verrillo E, et al. Night-to-night consistency of at-home nocturnal pulse oximetry testing for obstructive sleep apnea in children. Pediatr Pulmonol. 2013;48:754-60.

75. Meltzer $\sqcup$, Walsh CM, Traylor J, Westin AML. Direct comparison of two new actigraphs and polysomnography in children and adolescents. Sleep. 2012;35:159-66.

76. Toon E, Davey MJ, Hollis SL, et al. Comparison of commercial wrist-based and smartphone accelerometers, actigraphy, and PSG in a clinical cohort of children and adolescents. J Clin Sleep Med. 2016:12:343-50.

77. O'Driscoll DM, Foster AM, Davey MJ, et al. Can actigraphy measure sleep fragmentation in children? Arch Dis Child. 2010;95:1031.

78. Meltzer $\sqcup$, Hiruma LS, Avis K, et al. Comparison of a commercial accelerometer with polysomnography and actigraphy in children and adolescents. Sleep. 2015;38:1323-30.

79. Waltisberg D, Amft O, Brunner DP, Troster G. Detecting disordered breathing and limb movement using in-bed force sensors. IEEE $J$ Biomed Health Inform. 2017;21:930-8.
80. Park S, Shin $\mathrm{H}$. Feasibility study for unconstrained respiration monitoring based on multi-way approach using an acceleration and force sensing module. IEEE Sens J. 2017;17:3482-9.

81. Bronstein JZ, Brooks $\sqcup$. A potential alternative to respiratory inductance plethysmography for children? I Clin Sleep Med. 2016;13:159-60.

82. Mason DG, Iyer K, Terrill PI, et al. Pediatric obstructive sleep apnea assessment using pulse oximetry and dual RIP bands. Conf Proc IEEE Eng Med Biol SOC. 2010;2010:6154-7.

83. Griffiths AG, Patwari PP, Loghmanee DA, et al. Validation of polyvinylidene fluoride impedance sensor for respiratory event classification during polysomnography in children. J Clin Sleep Med. 2017;13:259-65.

84. Amaddeo A, Fernandez-Bolanos M, Olmo Arroyo J, et al. Validation of a suprasternal pressure sensor for sleep apnea classification in children. J Clin Sleep Med. 2016;12:1641-7.

85. Rofail LM, Wong KKH, Unger G, et al. The role of single-channel nasal airflow pressure transducer in the diagnosis of OSA in the sleep laboratory. J Clin Sleep Med. 2010;6:349-56.

86. Lesser DJ, Haddad GG, Bush RA, Pian MS. The utility of a portable recording device for screening of obstructive sleep apnea in obese adolescents. J Clin Sleep Med. 2012;8:271-7.

87. Gutiérrez-Tobal GC, Alonso-Álvarez ML, Álvarez D, et al. Diagnosis of pediatric obstructive sleep apnea: preliminary findings using automatic analysis of airflow and oximetry recordings obtained at patients' home. Biomed Signal Process Control. 2015;18:401-7.

88. Isaiah A, Pereira KD. Outcomes after adenotonsillectomy using a fixed anesthesia protocol in children with obstructive sleep apnea. Int J Pediatr Otorhinolaryngol. 2015;79:638-43.

89. Kheirandish-Gozal L, Gozal D. Pediatric OSA syndrome morbidity biomarkers: the hunt is finally on! Chest. 2017;151:500-6. 